\title{
Self-assembled micellar structures of Lipopeptides with variable number of attached lipid chains revealed by atomistic molecular dynamics simulations
}

Article

Accepted Version

Zhao, L., Tu, Y., Fang, H., Hamley, I. W. and Wang, Z. (2018) Self-assembled micellar structures of Lipopeptides with variable number of attached lipid chains revealed by atomistic molecular dynamics simulations. Journal of Physical Chemistry B, 122 (41). pp. 9605-9615. ISSN 1520-6106 doi: https://doi.org/10.1021/acs.jpcb.8b07877 Available at https://centaur.reading.ac.uk/79751/

It is advisable to refer to the publisher's version if you intend to cite from the work. See Guidance on citing.

To link to this article DOI: http://dx.doi.org/10.1021/acs.jpcb.8b07877

Publisher: American Chemical Society

All outputs in CentAUR are protected by Intellectual Property Rights law, including copyright law. Copyright and IPR is retained by the creators or other copyright holders. Terms and conditions for use of this material are defined in the End User Agreement. 


\section{www.reading.ac.uk/centaur}

\section{CentAUR}

Central Archive at the University of Reading

Reading's research outputs online 


\title{
Self-Assembled Micellar Structures of Lipopeptides with
}

\section{Variable Number of Attached Lipid Chains Revealed by}

\section{Atomistic Molecular Dynamics Simulations}

\author{
Liang Zhao ${ }^{\dagger}$, Yusong Tu* ${ }^{* \dagger}$, Haiping Fang», Ian W. Hamley ${ }^{\S}$ and Zuowei Wang*ף \\ ${ }^{\dagger}$ College of Physical Science and Technology, Yangzhou University, Jiangsu, 225009, China \\ Division of Interfacial Water and Key Laboratory of Interfacial Physics and Technology, Shanghai Institute of Applied Physics, \\ Chinese Academy of Sciences, Shanghai 201800, China \\ $\S_{\text {Department of Chemistry, University of Reading, Whiteknights, Reading, RG6 6AD, United Kingdom }}$ \\ ISchool of Mathematical, Physical and Computational Sciences, University of Reading, Whiteknights, Reading RG6 6AX, \\ United Kingdom
}

\begin{abstract}
We present atomistic molecular dynamics simulation study of the self-assembly behavior of toll-like agonist lipopeptides $\left(\mathrm{Pam}_{n} \mathrm{CSK} 4\right)$ in aqueous solutions. The variable number of hexadecyl lipid chains $(n=1,2,3)$ per molecule has been experimentally suggested to have remarkable influence on their self-assembled nanostructures. Starting from pre-assembled spherical or bilayer configurations, the aggregates of lipopeptides, PamCSK 4 and $\mathrm{Pam}_{2} \mathrm{CSK} 4$, which contain peptide sequences CSK4 linked to either mono- or di-lipid chains (Pam), evolve into sphericallike micelles within $30 \mathrm{~ns}$, whereas the self-assembled structure of tri-lipidated lipopeptides, $\operatorname{Pam}_{3} \mathrm{CSK} 4$, relaxes much slower and reaches an equilibrium state of flattened wormlike micelle with a bilayer packing structure. The geometric shapes and sizes, namely the gyration radii of spherical micelles and thickness of the flattened wormlike micelle, are found to be in good agreement with experimental measurements, which effectively validates the simulation models and employed force fields. Detailed analyses of molecular packing reveal that these self-assembled nanostructures all consist of a hydrophobic core constructed by lipid chains, a transitional layer and a hydrophilic interfacial layer composed of peptide sequences. The average area per peptide head at the interfaces is found to be nearly constant for all micellar structures studied. The packing parameter of the lipopeptide molecules thus increases with the increase of the number of linked lipid chains, giving rise to the distinct micellar shape transition from spherical-like to flattened wormlike geometry with bilayer stacking, which is qualitatively different from the shape transitions of surfactant micelles induced by variation of concentration or salt type. To facilitate the close-packing of the lipid chains in the hydrophobic core, the lipopeptide molecules typically take the bent conformation with average tilt angles between the peptide sequences and the lipid chains ranging from $110^{\circ}$ to $140^{\circ}$. This consequently affects the orientation angles of the lipid chains with respect to the radial or normal direction of the spherical-like or
\end{abstract}


flattened wormlike micelles. In addition, the secondary structures of the peptides may also be altered by the number of lipid chains they are linked to and the resultant micellar structures. Our simulation results on the microscopic structural features of the lipopeptide nanostructures may provide potential insights into their bioactivities and contribute to the design of bioactive medicines or drug carriers. The force fields built for these lipopeptides and the geometric packing discussions could also be adopted for simulating and understanding the self-assembly behavior of other bioactive amiphiphiles with similar chemical compositions.

\section{INTRODUCTION}

Lipopeptides, a class of bioactive amphiphilic molecules, have been widely used as antibacterial ingredients in human medicine for infections and immunity treatment, ${ }^{1,2}$ or as antifungal agents in crop storage to prevent spoilage. ${ }^{3}$ Lipopeptides consist of lipid chains connected to peptide sequences. The amphiphilic nature of these molecules favors the formation of aggregated structures in aqueous solutions owing to the low critical aggregation concentrations (cac), which leads to many applications in fabrication of biomedicines, ${ }^{4}$ design of drug carriers, ${ }^{5}$ as well as manufacture of imaging agents. ${ }^{6}$ The self-assembled structures typically take the forms of extended and rigid nanofibers or nanotapes, ${ }^{7-12}$ with some exceptions of wormlike micelles. ${ }^{13}$ The nanofibers can further construct 3-dimensional networks to provide structural support to collagen ${ }^{14}$ for reducing wrinkles and the appearance of aging of skin, reduce death of cells at the spinal cord injury sites, ${ }^{15}$ or stimulate/overcome host immune response. ${ }^{16}$

Recently Hamley and co-workers reported one of the very first experimental investigations on the self-assembly behavior of a class of toll-like receptor agonist lipopeptides, i.e., $\operatorname{Pam}_{n} \mathrm{CSK} 4(n=1,2,3)$, in aqueous solutions. ${ }^{17}$ These commerciallyavailable lipopeptides have shown bioactivity during the innate immune response. ${ }^{18}$ As sketched in Figure 1, the CSK4 peptides in these molecules are linked to different numbers of hexadecyl (palmitoyl, Pam) chains via glycerol units. Their selfassembled structures are suggested to be relevant to the binding specificity to the immune receptors. ${ }^{19}$ Using a combination of cryo-TEM, small-angle X-ray scattering and circular dichroism (CD) techniques, Hamley et al. observed the formation of distinct nanostructures: the mono- and di-lipidated molecules (PamCSK4 and $\mathrm{Pam}_{2} \mathrm{CSK} 4$ ) aggregate into spherical-like micelles, analogous to lipid surfactants, while unexpectedly the Pam ${ }_{3} \mathrm{CSK} 4$ molecules containing three lipid chains assemble into flexible flattened wormlike micelles with a bilayer packing. The cryo-TEM images of these self-assembled structures are shown in Figure 2. Molecular pictures on how these $\operatorname{Pam}_{n} \mathrm{CSK} 4$ molecules are packed into different structures have been proposed, which are however hard to verify at a molecular level. 


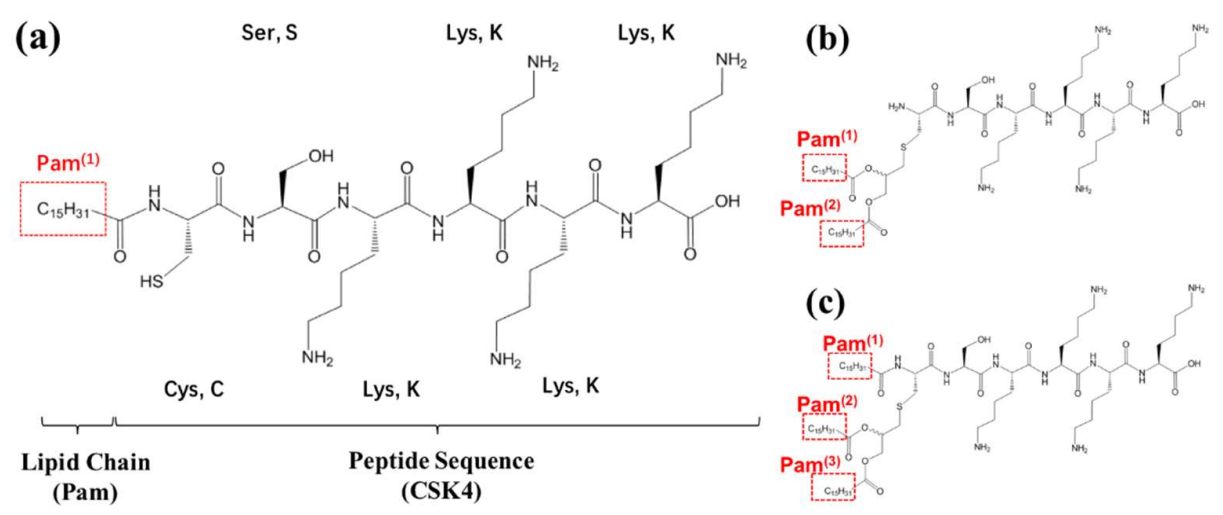

Figure 1. Molecular structures of $\mathrm{Pam}_{n} \mathrm{CSK} 4$ lipopeptides: (a) PamCSK4; (b) $\mathrm{Pam}_{2} \mathrm{CSK} 4$; (c) Pam ${ }_{3} \mathrm{CSK} 4$. These three kinds of lipopeptides contain different numbers of lipid chains (Palmitoyl, Pam) and the same peptide sequence (Cys-Ser-LysLys-Lys-Lys, CSKKKK). To distinguish the lipid chains in the same lipopeptide molecule, we label them as $\operatorname{Pam}^{(1)}$, $\operatorname{Pam}^{(2)}$ and $\operatorname{Pam}^{(3)}$, respectively.
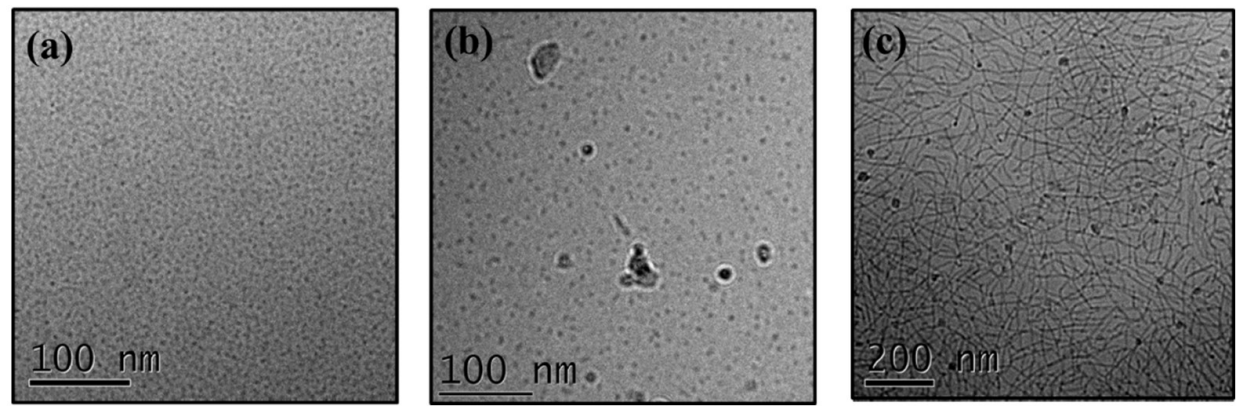

Figure 2. Cryo-TEM images of self-assembled structures of (a) PamCSK4, (b) $\mathrm{Pam}_{2} \mathrm{CSK} 4$ and (c) $\mathrm{Pam}_{3} \mathrm{CSK} 4$.

Computer simulations, including molecular dynamics (MD) and Monte Carlo (MC) simulations, allow the direct access to the atomistic details of molecules under investigation beyond the experimental resolution limit, ${ }^{20-25}$ and have become an effective tool to study the self-assembly process and the resultant structures. ${ }^{26}$ Surfactants are perhaps the most widely simulated class of amphiphiles, ${ }^{27-31}$ which have been described by models at various molecular levels using all-atom, united-atom or coarse-grained force fields. Depending on the system parameters, such as temperature, ionic strength and solute concentrations, ${ }^{32,33}$ self-assembled spherical-like or elongated micelles have been observed in aqueous solutions of surfactants. ${ }^{33-36}$ Regardless of their shapes and sizes, all micelles consist of a dry hydrophobic core surrounded by a disordered interfacial layer containing the headgroups, counterions, water molecules and methyl/methylene groups. The knowledge obtained from studying surfactant micelles provides a good reference for simulating the self-assembly behavior of more complex amphiphiles. For example, Schatz and co-workers performed atomistic simulations to determine the structural properties of cylindrical nanofibers formed by peptide amphiphiles consisting of a lipid chain attached to thirteen amino acids. ${ }^{37}$ They 
found a broad distribution of secondary structure of the peptide sequence in addition to the dry hydrophobic core and disordered interfacial region, which was consistent with experimental observations.

In this work, we perform atomistic MD simulations to investigate the self-assembled micellar structures formed by lipopeptides $\operatorname{Pam}_{n} \mathrm{CSK} 4$, in aqueous solutions. The converged configurations, including spherical-like micelles of PamCSK4 and $\mathrm{Pam}_{2} \mathrm{CSK} 4$ and flattened wormlike micelle of $\mathrm{Pam}_{3} \mathrm{CSK} 4$, are obtained by equilibrating the preassembled configurations after sufficiently long runs. The shapes and sizes of these equilibrated structures are found to be consistent with the experimental measurements. ${ }^{17}$ Detailed analyses of the arrangements of molecular packing inside the micelles, of the tilt angles between the peptide heads and lipid tails as well as the secondary structures of the peptide sequences are then carried out to provide microscopic structural features beyond the experimental resolution. A molecular model is also proposed to interpret the formation of these distinct self-assembled structures. These results on the self-assembled structures will contribute to the development of a microscopic understanding of the bioactivities of the lipopeptide micelles and so provide potential guidance for the design of bioactive medicines or drug carriers. To the best of our knowledge, this is the first atomistic simulation study of self-assembled structures of $\mathrm{Pam}_{n} \mathrm{CSK} 4$ lipopeptides, especially for the flattened wormlike micelle with a bilayer packing of $\mathrm{Pam}_{3} \mathrm{CSK} 4$. The force fields built for these lipopeptide molecules and the geometric packing model we proposed should also be applicable to simulate and understand the self-assembly behavior of a wide range of bioactive amphiphiles with similar chemical compositions.

\section{COMPUTATIONAL METHODS}

2.1. System Setup. As the spontaneous micellization of lipopeptides with long hydrophobic lipid tails and peptide heads from a random distribution is a timeconsuming process, we chose to use preassembled structures as the initial configurations, similar to previous studies of surfactant micelles ${ }^{34}$ and peptide amphiphile nanofibers. ${ }^{37}$ As shown in Figure 3a, the spherical-like micelle containing 45 PamCSK 4 molecules was extracted from a separate simulation of a preassembled aggregate comprising 80 PamCSK 4 molecules in aqueous solution which broke into two smaller stable micelles, one with 45 and the other with 35 molecules, respectively. The spherical-like micelle consisting of $76 \mathrm{Pam}_{2} \mathrm{CSK} 4$ molecules (Figure 3b) and the bilayer structure containing $180 \mathrm{Pam}_{3} \mathrm{CSK} 4$ molecules (Figure 3c) which extended infinitely in the y-direction due to periodic boundary condition were generated by the PACKMOL package. ${ }^{38}$ The aggregation numbers in the two spherical-like micelles are close to the experimental values of 36.3 for PamCSK 4 and 88.7 for Pam ${ }_{2}$ CSK 4 as estimated from the reported mean molecular volumes along with the volumes of the corresponding micelles based on a spherical close-packed picture. ${ }^{17}$

Each preassembled lipopeptide structure shown in Figure 3 was introduced into a simulation box whose dimensions are listed in Table 1 . The boxes were then filled with 
water molecules. Counterions (Chloride ions, $\mathrm{Cl}^{-}$) were also added to maintain neutralization of the systems. The concentrations of lipopeptides in all three systems, as measured in units of mass percentage, are far greater than their corresponding critical aggregation concentrations (cac). As a consequence, no lipopeptide molecules were found to dissociate from assembled configurations for the entire equilibrium simulation runs described below. More details about the system parameters are presented in Table 1.

(a)

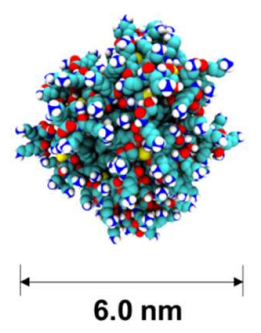

(b)

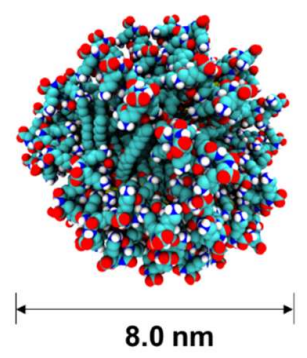

(c)

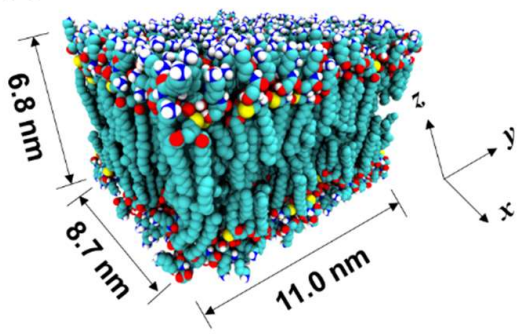

Figure 3. Snapshots of initial preassembled configurations of lipopeptides. (a) Spherical-like micelle containing 45 PamCSK4 molecules; (b) Spherical-like micelle containing $76 \mathrm{Pam}_{2} \mathrm{CSK} 4$ molecules placed along the radius direction; (c) Bilayer structure consisting of $180 \mathrm{Pam}_{3} \mathrm{CSK} 4$ molecules arranged in parallel, stacked into two layers with equal number of molecules and extending infinitely along the $y$ axis due to periodic boundary condition. Atoms are represented by VDW beads: carbon (cyan), oxygen (red), sulfur (yellow), nitrogen (blue) with bonded hydrogens (white), respectively. Water molecules and counterions are not shown for clarity.

Table 1. Numbers of Lipopeptides $\left(N_{l i p}\right)$, SPC water $\left(N_{w}\right)$ and Counterions $\left(N_{i o n}\right)$, Box Size $\left(V_{b o x}\right)$ and Mass Percentage $(w)$ of Lipopeptides in Each Simulated System.

\begin{tabular}{|c|c|c|c|c|c|}
\hline Lipopeptide & $N_{l i p}$ & $N_{w}$ & $N_{\text {ion }}$ & $V_{\text {box }}\left[\mathrm{nm}^{3}\right]$ & $w[\%]$ \\
\hline PamCSK4 & 45 & 53783 & 135 & $12.1 \times 12.1 \times 12.1$ & 4.26 \\
\hline $\mathrm{Pam}_{2} \mathrm{CSK} 4$ & 76 & 52927 & 304 & $12.0 \times 12.0 \times 12.0$ & 9.14 \\
\hline $\mathrm{Pam}_{3} \mathrm{CSK} 4$ & 180 & 36355 & 540 & $14.0 \times 11.0 \times 12.0$ & 28.8 \\
\hline
\end{tabular}

2.2. Simulation Details. All atomistic MD simulations were performed in the NPT ensemble using the GROMACS-4.5.4 software. ${ }^{39}$ Periodic boundary conditions were applied in all three directions. A constant temperature of $300 \mathrm{~K}$ and pressure of 1 bar were maintained by the $\mathrm{V}$-rescale and Parrinello-Rahman methods, ${ }^{40,41}$ with a coupling time of $0.1 \mathrm{ps}$ and of $1.5 \mathrm{ps}$, respectively. The SPC water model was used and its geometry was held by the SETTLE algorithm. ${ }^{42}$ No atomic coordinates were constrained during the simulations. The long-range electrostatic interactions were treated by the Particle-Mesh Ewald (PME) method with a real space cutoff radius of $1.2 \mathrm{~nm},{ }^{43,44}$ whereas the van der Waals interactions were truncated at a cutoff radius of $1.2 \mathrm{~nm}$. The GROMOS53A6 force field was used to parameterize the lipopeptides. ${ }^{45}$ 
The amino group $-\mathrm{NH}_{2}$ and the carboxyl group $-\mathrm{COOH}$ on lipopepides have been protonated and deprotonated into $-\mathrm{NH}_{3}(+)$ and $-\mathrm{COO}(-)$ groups in aqueous solution, with a unit positive and negative charge, respectively. The time step was 1.0 fs. Simulation data were collected for analyses every 1 ps during the last $10 \mathrm{~ns}$ after systems have reached their equilibrium states. Analyses using data collected from the last 20 ns provided nearly identical results within statistical errors.

\section{RESULTS AND DISCUSSION}

3.1. Equilibration of Preassembled Lipopeptide Nanostructures. The preassembled lipopeptide structures shown in Figure 3, especially those of $\mathrm{Pam}_{2} \mathrm{CSK} 4$ and $\mathrm{Pam}_{3} \mathrm{CSK} 4$, are unstable and should relax towards their equilibrium states by lowering the free energies. To track the equilibration process we plot the total nonbonded (electrostatic and van der Waals) energy for the whole micelle as a function of simulation time in Figure 4. We note that the long-range part of the electrostatic energy output by the g_energy command in GROMACS software is calculated for the whole system due to the use of the PME algorithm and it is difficult to decompose it into the contributions from each individual species. To better estimate the nonbonded energies of the lipopeptides, we have calculated the electrostatic interaction energies using the simple Coulomb's Law and the van der Waals energies using the Lennard-Jones pair potential among the lipopeptides themselves and between the lipopeptides and all other species in the system.

It is clear that the nonbonded energies of the PamCSK 4 and $\mathrm{Pam}_{2} \mathrm{CSK} 4$ micelles have converged to their plateau or equilibrium values within $30 \mathrm{~ns}$. The snapshots of the equilibrated micellar structures at $t=50 \mathrm{~ns}$ are shown in Figures $4 \mathrm{a}$ and $4 \mathrm{~b}$. The equilibration of the $\mathrm{Pam}_{3} \mathrm{CSK} 4$ structure is much slower, and the plateau-like value of the nonbonded energy is reached after about $150 \mathrm{~ns}$. The structural evolution of the system can be seen in Figure 4c from the representative snapshots taken at different simulation times. Starting from an initial bilayer stacking geometry, the Pam ${ }_{3} \mathrm{CSK} 4$ structure evolves into a hollow cylindrical wormlike micelle with a ring-like crosssection at $t=10 \mathrm{~ns}$ in order to reduce the exposure of the hydrophobic lipid tails to water. The hollow structure is still energetically unfavorable. As time further increases ( $t \geq 30 \mathrm{~ns}$ ), the cross-section of the wormlike micelle gradually changes its shape to diminish the vacuum void, which is accompanied by the slow decrease of the nonbonded energy of the self-assembled Pam ${ }_{3}$ CSK 4 structure. The snapshot taken at $t$ $=170 \mathrm{~ns}$ clearly reveals a flattened wormlike micellar structure with a bilayer packing of Pam ${ }_{3} \mathrm{CSK} 4$ molecules. This essentially verifies the molecular picture suggested by experiments. ${ }^{17}$

The structural analyses of the lipopeptide micelles in the following sections are all carried out in the equilibrium state, namely using the trajectories saved in the last $10 \mathrm{~ns}$ of each simulation run. 


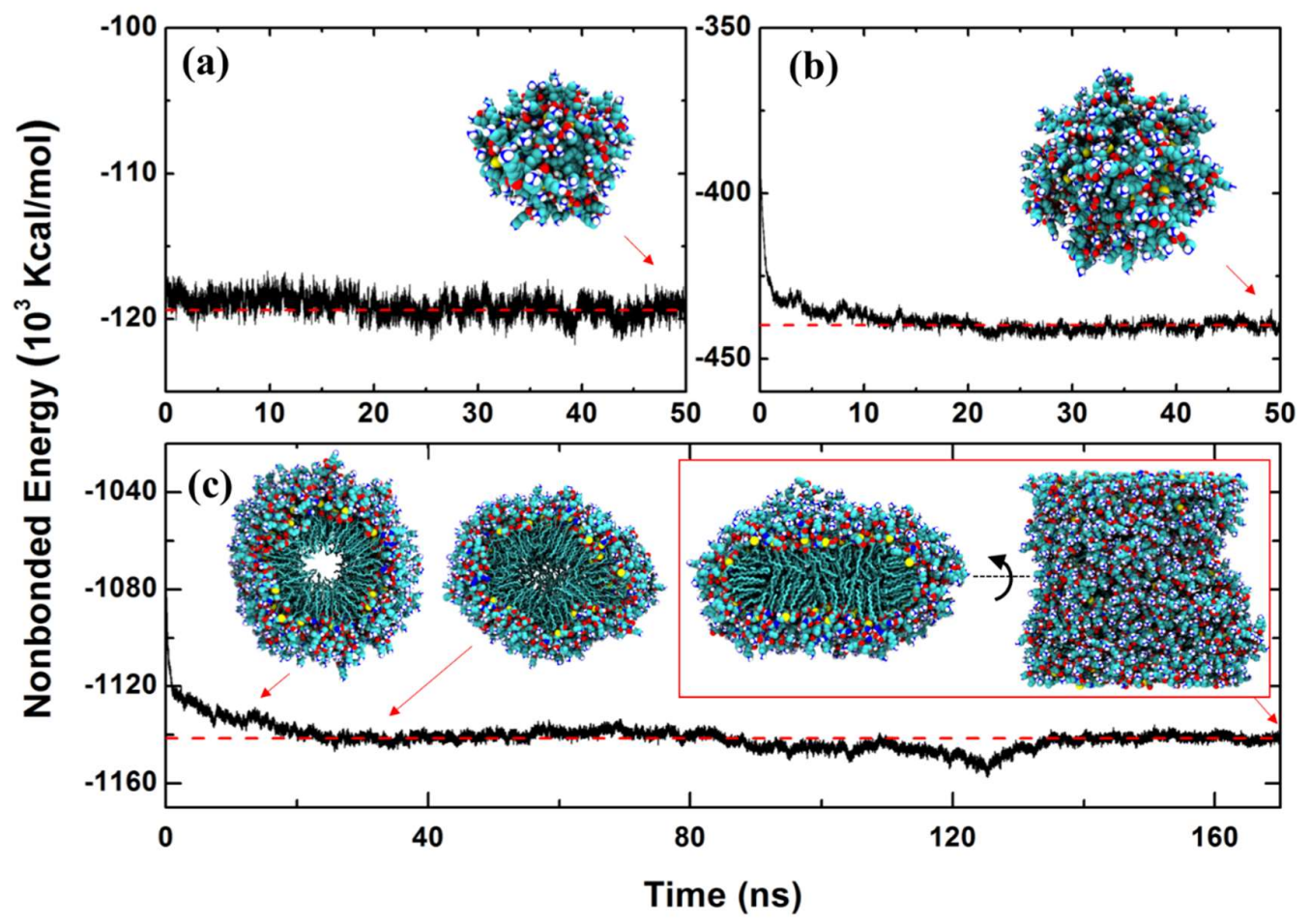

Figure 4. Nonbonded energies of self-assembled structures of (a) PamCSK4, (b) $\mathrm{Pam}_{2} \mathrm{CSK} 4$ and (c) Pam ${ }_{3}$ CSK 4 as a function of simulation time. The insets in (a) and (b) are snapshots of the corresponding spherical-like micelles taken at $t=50 \mathrm{~ns}$ when the systems have reached equilibrium state, while the snapshots in (c) are the crosssection view of the Pam ${ }_{3}$ CSK 4 wormlike micelle taken at $t=10,30$ and $170 \mathrm{ns,}$ respectively. The red dashed line shows the plateau value of the nonbonded energy of each micelle at the equilibrium state.

\subsection{Structural Features of Equilibrated Lipopetide Micelles.}

3.2.1. Geometric Shapes and Sizes. To make direct comparison with experimental measurements, ${ }^{17}$ we first analyze the geometric shapes and sizes of the equilibrated lipopeptide micelles. The geometric shape of a micellar structure with finite size can be characterized by the aspect ratios of the principal moments of inertia, $I_{i} / I_{j}$ with $i, j=1$, 2, 3, and the relative shape anisotropy $K^{2}$ as defined in PS. 1 in SI. ${ }^{46}$ The simulation results on $I_{i} / I_{j}$ and $K^{2}$ for the spherical-like micelles formed by PamCSK4 and $\mathrm{Pam}_{2} \mathrm{CSK} 4$ are presented in Table 2.

The sizes of the spherical-like lipopeptide micelles are measured by their radii of gyration, $R_{g}$, while the dimension of the infinitely long flattened wormlike micelle is characterized by the thickness $d$ of the bilayer region. Furthermore, we have also calculated the average volume, $v$, and average surface area, $a_{0}$, per lipopeptide in different structures. For the spherical-like micelles of PamCSK4 and Pam 2 CSK4 these quantities are estimated from the simple spherical geometry arguments that $v=$ $4 \pi R_{g}{ }^{3} / 3 N$ and $a_{0}=4 \pi R_{g}{ }^{2} / N$ where $N$ is the aggregation number. For the flattened 
wormlike micelle of $\mathrm{Pam}_{3} \mathrm{CSK} 4$, they are calculated in the bilayer region (excluding the curved side edges) using $v=w l d / N$ and $a_{0}=2 w l / N$, where $w$ denotes the width of the bilayer region and $N / l$ is the average number of $\mathrm{Pam}_{3} \mathrm{CSK} 4$ molecules per unit length, respectively. Table 3 lists the analysis results for $R_{g}, d, v$ and $a_{0}$, together with the average solvent accessible surface area per lipopeptide head, $a_{S A S A}$, for the three types of micelles.

Table 2. Geometric Shape Factors of Self-Assembled Structures of Lipopeptides: Aspect Ratios of Principal Moments of Inertia $I_{i} / I_{j}^{\mathrm{a}}$ and Relative Shape Anisotropy $K^{2}$.

\begin{tabular}{ccccc}
\hline Lipopeptide & $I_{1} / I_{3}$ & $I_{2} / I_{3}$ & $I_{1} / I_{2}$ & $K^{2}$ \\
\hline PamCSK4 & 0.73 & 0.93 & 0.78 & 0.04 \\
Pam $_{2}$ CSK4 & 0.78 & 0.93 & 0.85 & 0.02 \\
aPrincipal moments of inertia follow the sequence $I_{1}<I_{2}<I_{3}$. & & \\
\hline
\end{tabular}

Table 3. Size Factors of Self-Assembled Structures of Lipopeptides: Radius of Gyration $R_{g}$, Bilayer Thickness $d^{\text {a }}$, Average Volume $v$ and Surface Area $a_{0}$ Per Lipopeptide, Average Solvent Accessible Surface Area $a_{S A S A}$ Per Lipopeptide Head.

\begin{tabular}{ccccc}
\hline Lipopeptide & $R_{g}$ or $d(\mathrm{~nm})$ & $v\left(\mathrm{~nm}^{3}\right)$ & $a_{0}\left(\mathrm{~nm}^{2}\right)$ & $a_{\text {SASA }}\left(\mathrm{nm}^{2}\right)$ \\
\hline PamCSK4 & $2.16\left(R_{g}\right)$ & 0.94 & 1.30 & 5.66 \\
Pam 2 CSK4 & $3.01\left(R_{g}\right)$ & 1.50 & 1.50 & 8.70 \\
Pam 3 CSK4 & $5.0(d)$ & 3.26 & 1.30 & 9.86
\end{tabular}

${ }^{a}$ Thickness of the flat bilayer region. The radius of gyration is not the proper quantity to describe the scale of the bilayer structure because of its large deviation from spherical shape.

The aspect ratios of the principal moments of interia, $I_{i} / I_{j}(0.73-0.93)$, indicate that the micellar structures formed by the PamCSK 4 and $\mathrm{Pam}_{2} \mathrm{CSK} 4$ molecules are of modestly ellipsoidal shape. This is also confirmed by the small values of the relative shape anisotropy, i.e., $K^{2}=0.04$ for PamCSK 4 and 0.02 for Pam ${ }_{2} \mathrm{CSK} 4$, which are close to 0 as expected for a perfect sphere. It is thus reasonable to call the self-assembled structures of PamCSK 4 and $\mathrm{Pam}_{2} \mathrm{CSK} 4$ spherical-like micelles to distinguish from the flattened wormlike micelle formed by $\mathrm{Pam}_{3} \mathrm{CSK} 4$. The gyration radii of the simulated PamCSK4 and Pam ${ }_{2}$ CSK 4 micelles are found to be $R_{g}=2.16 \mathrm{~nm}$ and $3.01 \mathrm{~nm}$, respectively. These values are in good agreement with the experimental results of 2.2 $\mathrm{nm}$ and $3.3 \mathrm{~nm}$ obtained from SAXS. ${ }^{17}$

For the flattened wormlike micelle of Pam ${ }_{3}$ CSK 4 with a bilayer cross-section (see Figure $4 \mathrm{c}$ for the snapshot taken at $t=170 \mathrm{~ns}$ ) the thickness of the bilayer region can be estimated from the atomic density functions as described in the following subsection and found to be about $5.0 \mathrm{~nm}$, which is again consistent with the experimental value of $5.2 \mathrm{~nm} .{ }^{17}$

Table 3 shows that the average volume $v$ per lipopeptide molecule increases with the increase of the number of lipid tails, as expected. The surface area $a_{0}$ per lipopeptide is however almost constant in the three different types of micelles which can be attributed 
to the presence of the same peptide sequence head CSK4 in the lipopeptides studied. The situation is different from the self-assembled structures formed by the same type of surfactants where the value of $a_{0}$ decreases as the aggregate shape changes from spherical to wormlike micelle and then to lamellar phase with increasing surfactant density. ${ }^{32,47}$ On the other hand, the average solvent accessible surface area $a_{S A S A}$ per lipopeptide head is found to increase with the number of lipid tails per molecule. This can be understood from the bending or torsion of peptide sequences in the micellar structure, as discussed in more detail below.

The good agreement between the simulation and experimental results on the sizes and geometric properties of the lipopeptide micelles effectively validates the simulation system setups and force fields we employed. Further analyses can then be performed to provide information at the atomic level on the structural features of these distinct nanostructures and their dependence on the molecular architectures of the lipopeptides.

3.2.2. Molecular Packing Inside Micelles. To examine the packing details of lipopeptides inside the self-assembled structures, we calculate the atomic density functions (ADFs) of selected components either as functions of their radial distances from the centers of mass (COMs) of the spherical-like micelles, or as functions of their normal distances from the midplane of the bilayer region of the flattened wormlike micelle.
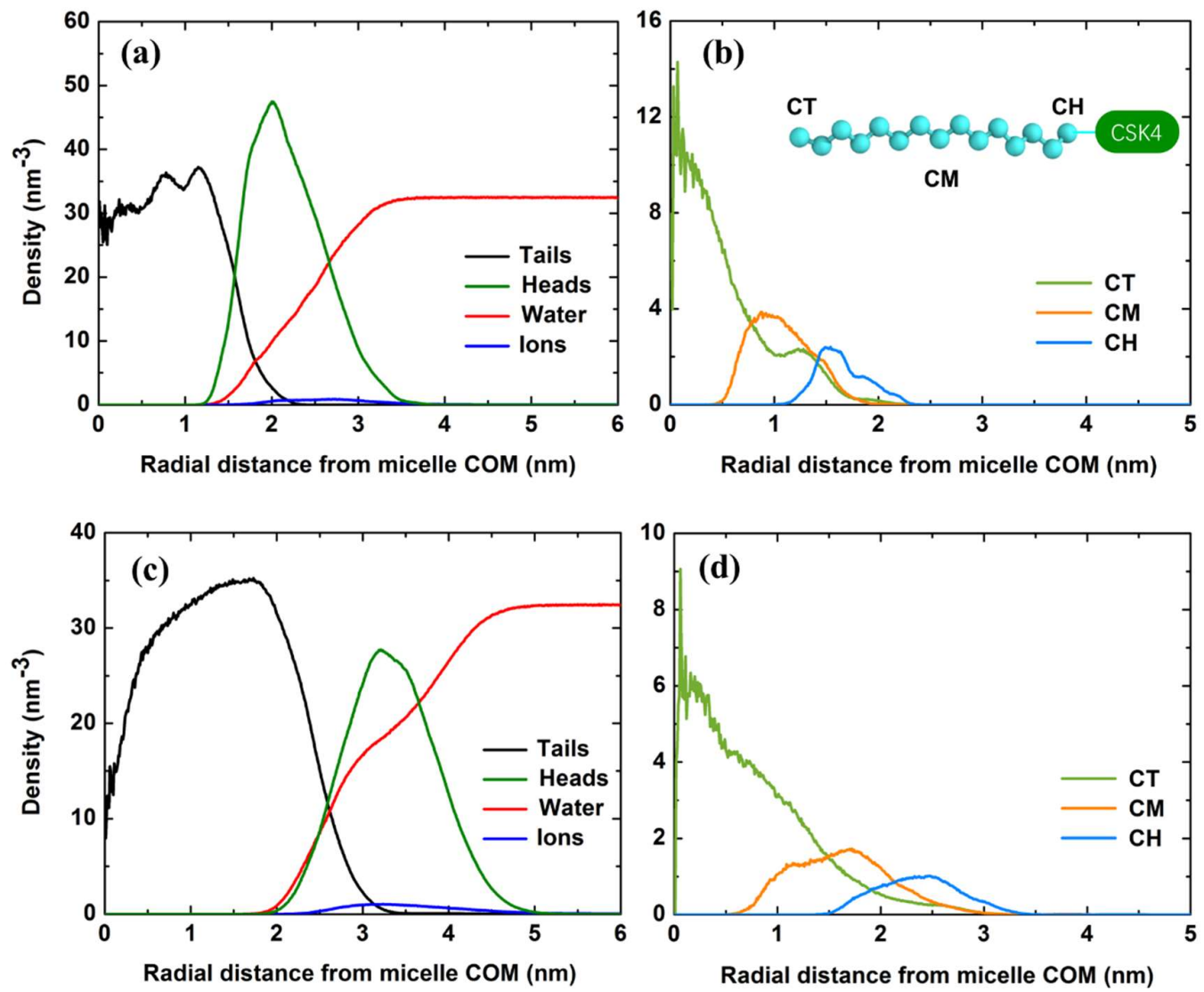

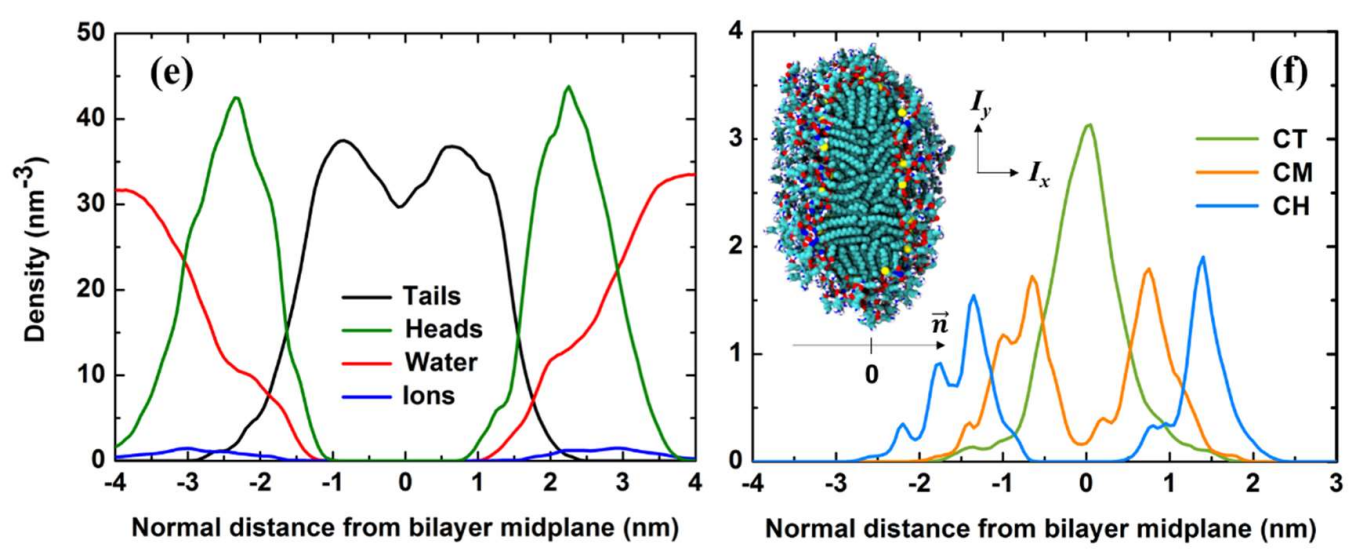

Figure 5. Atomic density functions for the spherical-like micelles of $(a, b)$ PamCSK4 and (c, d) $\mathrm{Pam}_{2} \mathrm{CSK} 4$, and for the flattened wormlike micelle of $(\mathrm{e}, \mathrm{f}) \mathrm{Pam}_{3} \mathrm{CSK} 4$. Left panels: ADFs divided into lipid tail, peptide head, water and ion contributions with respect to the center of mass $(\mathrm{COM})$ of the spherical-like micelle, or along the normal direction of the flattened wormlike micelle (see inset of (f)). The normal vector is identified by the principal axes of the inertia tensor. To clearly show the stacking structure within the flattened wormlike micelle of Pam ${ }_{3} \mathrm{CSK} 4$, only molecules in the central $4 \mathrm{~nm}$ width region in the $I_{y}$ direction are considered. Right panels: ADFs of the head and middle methylene groups and the terminal methyl groups, denoted as " $\mathrm{CH}$ ", "CM" and "CT", on lipid chains. The inset in (b) gives the schematic representation of the positions of "CH", "CM" and "CT" on a lipid chain. The normal vector of the flattened wormlike micelle is shown in the inset of (f). Only one lipid chain in each lipopeptide molecule is considered in the calculation of $(b, d, f)$, namely Pam $^{(1)}$ on PamCSK4, $\mathrm{Pam}^{(2)}$ on $\mathrm{Pam}_{2} \mathrm{CSK} 4$ and $\mathrm{Pam}^{(3)}$ on Pam 3 CSK4 (see Figure 1).

The ADFs in Figure 5a show that the spherical-like micelle of PamCSK4 consists of a hydrophobic core containing only lipid chains, which extends to about $1.2 \mathrm{~nm}$ from the micelle center. From there outward, we can see a transitional region containing a mixture of methyl/methylene groups from the lipid tails, amide groups from the peptide sequences, counterions and water molecules. This Stern layer can extend to about 2.0 $\mathrm{nm}$. The outermost layer of the micelle is a rough interface including amino acids, counterions and water. The density of water increases rapidly away from the COM of the micelle and reaches the bulk value $\sim 33 \mathrm{~nm}^{-3}$ at the radial distance of about $3.5 \mathrm{~nm}$. Similar atomic distributions along the radial direction also exist in the spherical-like micelle of $\mathrm{Pam}_{2} \mathrm{CSK} 4$. There the radius of the hydrophobic core is about $1.7 \mathrm{~nm}$, followed by a Stern layer about $1.8 \mathrm{~nm}$ thick. The interfacial region spans from 3.5 to $5.0 \mathrm{~nm}$.

Figures $5 \mathrm{~b}$ and $5 \mathrm{~d}$ show the density distributions of selected methyl/methylene groups on the lipid chains in the spherical-like micelles of PamCSK 4 and $\mathrm{Pam}_{2} \mathrm{CSK} 4$, respectively. For simplicity, only one lipid chain from each lipopeptide molecule, namely $\mathrm{Pam}^{(1)}$ for PamCSK4 and $\mathrm{Pam}^{(2)}$ for Pam 2 CSK4, is considered in the calculations. The ADFs of $\mathrm{Pam}^{(1)}$ chains on the Pam 2 CSK 4 molecules are very similar to those of $\mathrm{Pam}^{(2)}$ on the same molecules, see Figure S1 in Supporting Information (SI). 
In both micelles, the ADFs of the terminal "CT" groups are peaked around the micelle centers and decay quickly outwards, indicating that the terminals of the lipid chains are hidden deep inside the micellar cores. The distributions and their peak positions of the middle "CM" and head "CH" groups shift away from the micelle COM, implying a radial arrangement of the lipid chains in the micellar cores which can be further analyzed from the orientations of these hydrophobic chains.

As sketched in the insets of Figures $6 \mathrm{a}$ and $6 \mathrm{~b}$, the orientation of a lipid chain in a spherical-like micelle is defined by the angle $\theta$ between its end-to-end vector pointing from the head methylene group " $\mathrm{CH}$ " to the terminal methyl group "CT" and the radial vector pointing from the head group " $\mathrm{CH}$ " to the $\mathrm{COM}$ of the micelle. When the value of $\theta$ is $0^{\circ}$, the lipid chain is perfectly aligned along the radial direction of the micelle. ${ }^{48}$ However, due to thermal fluctuations in the chain conformation and orientation as well as the fact that the number of lipid chains in an actual micelle could be different from that required to construct an ideal sphere with radius equal to the mean end-to-end distance of the chains, the orientation angles have a relatively broad probability distribution, as shown in Figure 6a for the PamCSK4 micelle. In this case, the number of Pam chains $(N=45)$ is larger than that estimated for a spherical micelle $(N=36.3)$, the simulated micelle thus takes a slightly elongated shape, see Table 2 . The orientations of some lipid chains are more in alignment with the radial direction than the others, resulting in two peaks located around $20^{\circ}$ and $45^{\circ}$ in the probability distribution. This might be related to the bent conformation of the PamCSK4 molecules as discussed in Sec. 3.3.

Each $\mathrm{Pam}_{2} \mathrm{CSK} 4$ has two lipid chains, $\mathrm{Pam}^{(1)}$ and $\mathrm{Pam}^{(2)}$, which are chemically equivalent in molecular structure as sketched in Figure 1b. Inside the spherical-like micelle, their orientation angles with respect to the radial direction show very similar probability distributions with a peak located at about $20^{\circ}$ (Figure 6b). This similarity reflects the statistical equivalency of the two lipid chains in the micellar core undergoing thermal fluctuations. The indistinguishable feature of these two chains can also be seen from the inset plot of Figure $6 \mathrm{~b}$ where the angle between the end-to-end vectors of two lipid chains in the same molecule fluctuates over a range close to that of the radial orientation angle $\theta$. In both of the PamCSK4 and Pam ${ }_{2} \mathrm{CSK} 4$ micelles, the lipid chains are stiff and have the end-to-end lengths of about $1.5 \mathrm{~nm}$ (Figure S2 in SI). The higher peak at $\theta \approx 20^{\circ}$ in the $\mathrm{Pam}_{2} \mathrm{CSK} 4$ case indicates that the lipid chains more preferably align along the radial direction than those in the PamCSK4 case. This may be related to the smaller number of $\mathrm{Pam}_{2} \mathrm{CSK} 4$ molecules $(N=76)$ than that estimated for an ideal spherical micelle $(N=88.6)$, which gives more space for the lipid chains to take their preferred orientations. As will be seen below, the orientations of the lipid chains in the hydrophobic core may also be correlated with the arrangements of the linked peptide sequences in the interfacial layer.

Different from the spherical-like micelles formed by lipopeptides with mono- and dilipid chains, the ADFs in the flattened wormlike micelle of Pam ${ }_{3} \mathrm{CSK} 4$ (Figure 5e) demonstrates the symmetric distributions of lipid chains, peptide sequences, water and counterions about the midplane of the micelle. Distribution functions of selected methyl/methylene groups on the lipid chains $\left(\mathrm{Pam}^{(3)}\right)$ in Figure 5f show similar 
symmetric behavior. The symmetry in the atomic distributions further verifies the formation of a bilayer stacking structure that consists of a central hydrophobic region about $2 \mathrm{~nm}$ thick, a Stern layer and an interfacial layer in direct contact with water. The overall thickness of the flattened wormlike micelle is measured by the distance between the two peaks in the ADF of the peptide sequences in Figure 5e and found to be $5 \mathrm{~nm}$ as mentioned above.
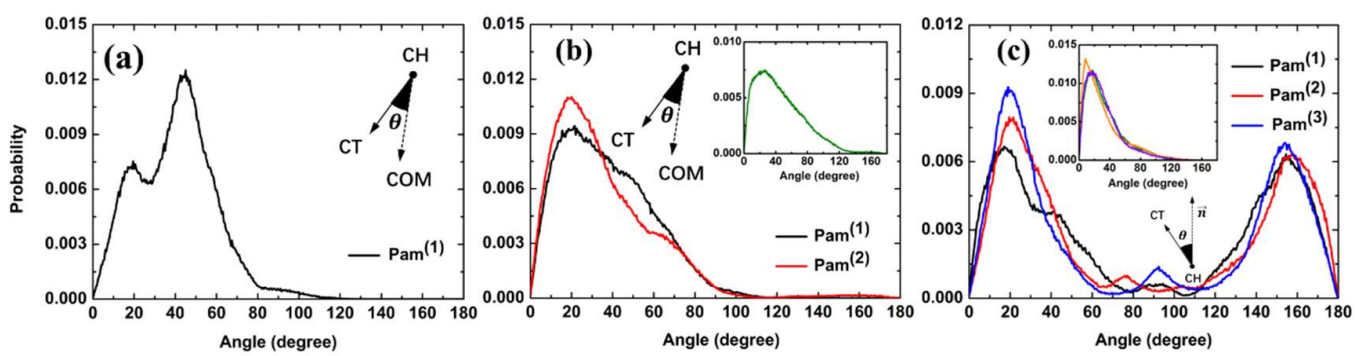

Figure 6. Probability distributions of angles between the end-to-end vectors of lipid chains and the radial vector of spherical micelles of (a) PamCSK4 and (b) Pam 2 CSK4 or the normal vector of the flattened wormlike micelle of (c) Pam ${ }_{3}$ CSK4. The insets show the probability distributions of the angle between the end-to-end vectors of any two lipid chains on the same molecule. In (c) only Pam ${ }_{3}$ CSK 4 molecules in the $4 \mathrm{~nm}$ wide region along the $I_{y}$ direction of flattened wormlike micelle are considered in order to show clearly the bilayer packing of the lipid chains.

The orientation of a lipid chain in the flattened wormlike micelle of Pam ${ }_{3}$ CSK 4 is defined by the angle $\theta$ between its end-to-end vector and the normal vector of the midplane of the micelle, see the sketch in Figure $6 \mathrm{c}$. When this angle is $0^{\circ}$ or $180^{\circ}$, the lipid chain is perfectly aligned in the normal direction. Figure $6 \mathrm{c}$ shows the probability distributions of the orientation angles of the three lipid chains, $\mathrm{Pam}^{(1)}, \mathrm{Pam}^{(2)}$ and $\mathrm{Pam}^{(3)}$ (Figure 1c), of $\mathrm{Pam}_{3} \mathrm{CSK} 4$, which are again very close to each other due to the statistical indistinguishability of these chains in the hydrophobic region. This similarity is further supported by the nearly identical distributions of the angles between any pairs of Pam chains in the same Pam ${ }_{3} \mathrm{CSK} 4$ molecule (inset plot of Figure 6c). We note that the results in Figure 6c are obtained by excluding the Pam ${ }_{3} \mathrm{CSK} 4$ molecules at the two curved edges of the micelle, because their orientation angles mainly contribute to the distributions around $\theta \approx 90^{\circ}$ (Figure S3 in SI) and so have little impact on the discussions of the important bilayer features. In comparison with the results in Figure $6 \mathrm{a}$ and $6 \mathrm{~b}$, the orientation angles of lipid chains in the flattened wormlike micelle show narrower distributions peaked at $\theta \approx 20^{\circ}$ and $160^{\circ}$ with approximately equal probability. The somewhat tilted alignment of the lipid chains in the bilayer region can be directly visualized in the snapshot of the cross-section of the micelle in Figure $4 \mathrm{c}$ at $t=170 \mathrm{~ns}$. This configuration can well explain the result that the effective thickness of the hydrophobic region $(2 \mathrm{~nm})$ is smaller than twice the lipid chain length $(2 \times 1.5 \mathrm{~nm}=3$ $\mathrm{nm})$. 
3.2.3. Geometric Interpretation of Distinct Micellar Structure Formation. The distinct nanostructures formed by lipopeptides containing the same peptide head but different numbers of lipid tails can be understood from a simple geometric argument. Israelachvili suggested ${ }^{49}$ that the geometric shape of a nanostructure assembled by amphiphilic molecules can be predicted by the value of a dimensionless packing parameter, $P=v_{\text {tail }} /\left(a_{0} l_{c}\right)$, where $a_{0}$ is the average area per molecule at the aggregate interface, $v_{\text {tail }}$ is the total volume of hydrophobic tails in one molecule and $l_{c}$ is the critical length of the hydrophobic tails. For a lipopeptide molecule with $n$ lipid chains, $v_{\text {tail }}$ can be roughly estimated as $n S_{0} l_{c}$ where $S_{0}$ is the cross-sectional area of a lipid chain and the $n$ lipid chains are assumed to orient in parallel to form a bundle-like structure inside the hydrophobic core. The packing parameter is then given by $P \approx n S_{0} / a_{0}$. If $P \approx$ 1 , the lipopeptides are of cylindrical shape due to nearly equal head and tail crosssections and tend to aggregate in parallel to from bilayer or lamellar structure. If $P<1$, the lipopeptides effectively have a cone-like shape and so prefer to form spherical-like micelles.

The radius of the cross-section of a lipid chain, $r$, can be simply estimated as half of the average distance in between pairs of the head " $\mathrm{CH}$ " groups of the three lipid chains in a Pam ${ }_{3} \mathrm{CSK} 4$ molecule and is found to be $0.36 \mathrm{~nm}$. The corresponding average crosssection area is then $S_{0} \approx \pi r^{2} \sim 0.41 \mathrm{~nm}^{2}$. The average surface area per molecule is given in Table 3 as $a_{0} \sim 1.3 \mathrm{~nm}^{2}$ for all three types of lipopeptides. It follows that the packing parameter is $P=S_{0} / a_{0} \sim 0.32$ for PamCSK4, which is close to the theoretical value of $P=1 / 3$ predicted for ideal spherical micelle formed by single-tail surfactants and $P=$ $2 S_{0} / a_{0} \sim 0.63$ for $\mathrm{Pam}_{2} \mathrm{CSK} 4$, indicating the preference for these lipopeptides to selfassemble into spherical-like micelles, as observed in experiments ${ }^{17}$ and our simulations. On the other hand, the packing parameter for Pam ${ }_{3}$ CSK 4 is $P=3 S_{0} / a_{0} \sim 0.95$ that is very close to 1.0, which explains the bilayer stacking structure in the flattened wormlike micelle.

The formation of distinct nanostructures, from spherical-like micelles to flattened wormlike micelle with a bilayer packing, is thus caused by the increase in the number of lipid chains per lipopeptide, which effectively changes the geometric shape of individual molecules and consequently alters their packing behavior inside the micelles. This mechanism is qualitatively different from the micellar shape transition observed in aqueous solutions of surfactants with constant number of hydrophobic tails, which is driven by the increase of the surfactant concentration ${ }^{32,47}$ or change in salt type or concentration. ${ }^{34}$

3.3. Structural Features of Peptides in Interfacial Layer. The bioactivities of the self-assembled lipopeptide nanostructures are predominantly controlled by the structural features of peptides in the interfacial layers. The Pam ${ }_{n} \mathrm{CSK} 4$ molecules studied in this work all contain the same CSK4 peptide sequence. As shown in Table 3, peptides linked to different numbers of lipid chains tend to occupy the same average area per head at the micelle interfaces. In order to facilitate the close-packing of the lipid chains in the hydrophobic core, the lipopeptides need to take bent conformations 
with various tilt angles between the peptide heads and the lipid tails. This will consequently affect the orientation angles of the lipid chains in the micellar core as discussed in the previous section. In addition, the secondary structures of the peptides may also be altered by the number of lipid chains they are linked to and the resultant micellar structures.

3.3.1. Tilt Angle Between Peptide Head and Lipid Chains. The tilt angle $\varphi$ between the peptide head and a lipid chain in a $\operatorname{Pam}_{n} \mathrm{CSK} 4$ molecule is defined as the angle between the end-to-end vector of the CSK4 peptide, pointing from the nitrogen atom of the $-\mathrm{NH}_{2}(-\mathrm{NH}-)$ group on the cysteine to the carbon atom of the $-\mathrm{COOH}$ group on the lysine, and the end-to-end vector of the lipid chain. By this convention, a lipopeptide molecule is fully extended if the tilt angle $\varphi=180^{\circ}$.
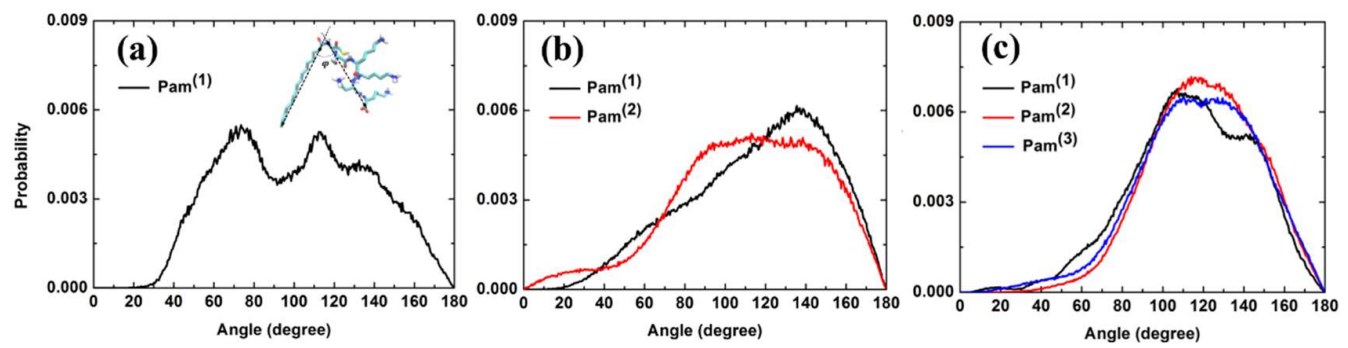

Figure 7. Probability distributions of tilt angles between peptide head and different lipid chains in (a) PamCSK4 and (b) $\mathrm{Pam}_{2} \mathrm{CSK} 4$ molecules in their corresponding spherical-like micelles, and in (c) $\mathrm{Pam}_{3} \mathrm{CSK} 4$ molecules in the flattened wormlike micelle.

The PamCSK 4 molecule has the smallest packing parameter $(\sim 0.32)$. As shown in Figure 7a, the tilt angles of these molecules in the spherical-like micelle have a broad distribution spanning from $30^{\circ}$ to $180^{\circ}$, indicating that the PamCSK4 molecules in the micelle are far from fully extended but instead have a strongly bent conformation with the tilt angle fluctuating about the average value of $\sim 100^{\circ}$. This type of bent conformation was not considered in the original geometric packing description of the micellar shape. ${ }^{49}$ To understand the two peaks in the probability distribution of the orientation angles of the lipid chains in Figure $6 \mathrm{a}$, we may refer to the two peaks in the tilt angle distribution at $72^{\circ}$ and $113^{\circ}$ in Figure $7 \mathrm{a}$. It suggests that the PamCSK4 molecules with more extended conformations (higher $\varphi$ values) are in better alignment with the micellar radial direction than the more bent ones. This is evidenced in Figure 8 where the peak of the probability distribution of the tilt angles of the PamCSK 4 molecules whose lipid chains are at smaller orientation angles $\left(\theta=20^{\circ} \pm 5^{\circ}\right)$ in the micelle is located at higher $\varphi$ value than that of the PamCSK4 molecules with larger orientation angle $\left(\theta=45^{\circ} \pm 5^{\circ}\right)$. 


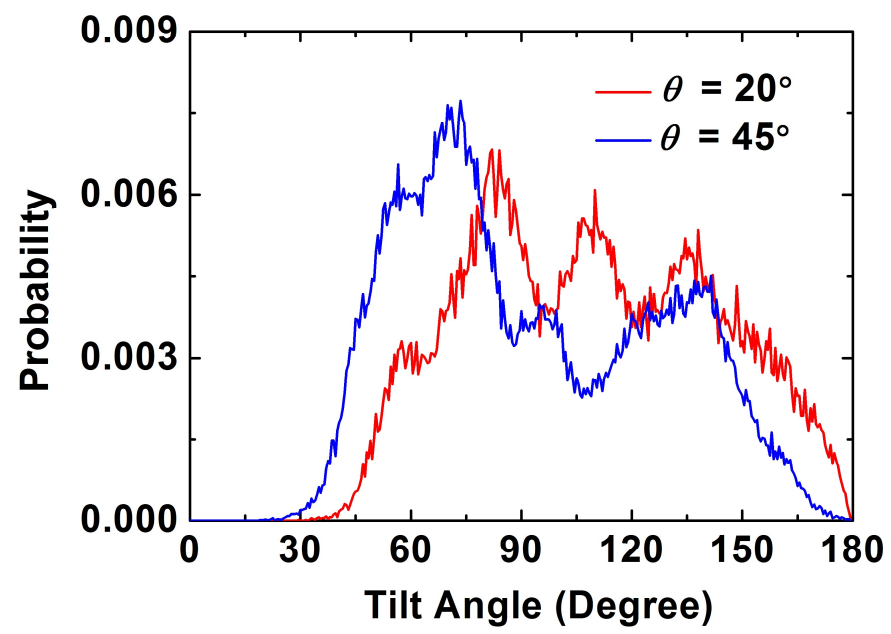

Figure 8. Probability distributions of tilt angles $\varphi$ of PamCSK 4 molecules whose lipid chains are at the two most probable orientation angles $\theta=20^{\circ} \pm 5^{\circ}$ and $45^{\circ} \pm 5^{\circ}$ in the micelle (see Figure 6a).

Comparing Figures $7 \mathrm{~b}$ and $7 \mathrm{c}$ with Figure $7 \mathrm{a}$, we can see that with an increase of the number of lipid chains per molecule, the peak of the probability distribution of the tilt angles shifts to larger $\varphi$ values. In Figure 7b, the distributions of the tilt angles between the peptide head and the two linked lipid chains in the Pam 2 CSK 4 molecules are similar to each other, with some small differences in the plateau-like region from about $100^{\circ}$ to $140^{\circ}$ for the $\mathrm{Pam}^{(2)}$ chains and a single peak at $140^{\circ}$ for the Pam ${ }^{(1)}$ chains, both reflecting a more extended conformation of $\mathrm{Pam}_{2} \mathrm{CSK} 4$ than PamCSK4. For $\mathrm{Pam}_{3}$ CSK 4 molecules in the flattened wormlike micelle, the tilt angles between the peptide head and the three linked lipid chains follow very similar distributions with a plateau-like region from $110^{\circ}$ to $140^{\circ}$, an indication of not fully extended conformation. This similarity is consistent with the orientation angle distributions of the lipid chains shown in Figure $6 c$ and further confirms their statistical equivalency.

The tilting of the peptide head with respect to the linked lipid chains exists in all selfassembled structures of mono-, di- and tri-lipidated lipopeptides we studied. Such bent conformations not only alter the interactions and packing arrangements of lipopeptide molecules within the micelles, but also increase the number of charged groups in the peptides exposed to the surrounding aqueous environment. The latter effect may facilitate the adsorption of water molecules and ions on the micellar surface, and enhance the contact of peptide sequences with other biological molecules, such as proteins and DNA.

3.3.2. Secondary Structures of Peptide Sequences. Based on calculations using the STRIDE software, ${ }^{50}$ we find that the peptide sequences of lipopeptide molecules in the self-assembled nanostructures exhibit various secondary structures, including $\beta$-sheet, $\beta$-turn and random coil configurations. Table 4 lists the average populations in the three micellar systems we studied. The $\beta$-turn and random coil configurations dominate the 
secondary structures of peptides in all of the self-assembled lipopeptides with their total population up to $99 \%$. The PamCSK 4 and $\mathrm{Pam}_{2} \mathrm{CSK} 4$ molecules in the spherical-like micelles show rather similar population proportions of $\beta$-turn $(>80 \%)$ and random coil $(<20 \%)$ configurations, but for $\mathrm{Pam}_{3} \mathrm{CSK} 4$ in the flattened wormlike micelle the population of $\beta$-turn decreases to $67.8 \%$ and that of random coil increases to $31.1 \%$. The $\beta$-sheet shows small population $(\sim 1.0 \%)$ in all of the self-assembled structures. These simulation results seem to imply that the peptide sequences tend to take disordered configurations and they are more randomly coiled in the flattened worm micelles of $\mathrm{Pam}_{3} \mathrm{CSK} 4$. However, according to the circular dichroism spectra reported by Hamley et al., ${ }^{17}$ the $\beta$-sheet configuration should be the dominant peptide secondary structure in the flattened worm micelles of $\mathrm{Pam}_{3} \mathrm{CSK} 4$ at the experimental temperature of $300 \mathrm{~K}$. This inconsistency presumably results from the difference between the experimental and simulation temperatures. As inferred from the simulations of phase transition of bilayer membrane, ${ }^{51}$ the secondary structures obtained at the simulation temperature of $300 \mathrm{~K}$ in fact reflect the configurations at the experimental temperature $\sim 320 \mathrm{~K}$. Indeed, the secondary structures of peptide sequences in the self-assembled structures of $\mathrm{Pam}_{3} \mathrm{CSK} 4$ at temperature of $320 \mathrm{~K}$ show a disordered configuration. ${ }^{17}$ Since the main focus of the current work is on the distinct self-assembled structures of lipopeptides and their dependence on the number of lipid chains per molecule, we will leave more detailed studies on the secondary structures of peptide sequences for future simulation work.

Table 4. Average Populations of Secondary Structures of CSK4 Peptide Sequences in Three Kinds of Self-Assembled Structures of Lipopeptides Simulated at $300 \mathrm{~K}$.

\begin{tabular}{cccc}
\hline Lipopeptide & $\beta$-Sheet $(\%)$ & $\beta$-Turn $(\%)$ & Random Coil (\%) \\
\hline PamCSK4 & 0.4 & 80.2 & 19.4 \\
Pam 2 CSK4 & 0.7 & 84.8 & 14.5 \\
Pam $_{3}$ CSK4 & 1.1 & 67.8 & 31.1 \\
\hline
\end{tabular}

\section{CONCLUSIONS}

Atomistic molecular dynamics simulations are performed to study the self-assembly behavior of lipopeptides, $\operatorname{Pam}_{n}$ CSK 4 which contain the same peptide sequence CSK 4 but different number of lipid chains (Pam), in aqueous solutions. All simulations start from preassembled initial configurations and run long enough until the potential energies of the aggregates drop to the plateau regime. In the final (equilibrated) state, the mono-lipidated (PamCSK4) and di-lipidated (Pam 2 CSK4) lipopeptides are found to form spherical-like micelles with radii of gyration of about 2.2 and $3.3 \mathrm{~nm}$, respectively. In clear contrast, the tri-lipidated lipopeptide, $\mathrm{Pam}_{3} \mathrm{CSK} 4$, self-assembles into flattened wormlike micelles with a bilayer stacking that is about $5.0 \mathrm{~nm}$ in thickness. The geometric shapes and sizes of the simulated micellar structures are in good agreement with those observed in experiments. This effectively validates the 
simulation models and force fields we employed, and so allows for further structural analyses. In analogy to surfactant micelles, the lipopeptide micelles consist of a hydrophobic core constructed by lipid chains, a transitional region containing a mixture of methyl/methylene groups from the lipid tails, amide acids from the peptide sequences, counterions and water molecules, and a hydrophilic interfacial layer of peptide sequences. Lipid chains in the micellar cores are about $1.5 \mathrm{~nm}$ in length without torsion. Their orientation angles with respect to the radial direction of the spherical-like micelles or the normal direction of the bilayer region of the flattened wormlike micelle typically deviate from the perfect alignment by $20^{\circ}$ to $45^{\circ}$ with the broadest distribution found in the micelle of PamCSK4.

In all of the lipopeptide micelles we simulated, the average surface area, $a_{0}$, per peptide head is nearly constant, regardless of the number of lipid chains connected. As a consequence, the packing parameter, which can be written as $P=n S_{0} / a_{0}$ with $S_{0}$ being the cross-section area of a lipid chain, increases from $\sim 0.32$ to about 1 with the increase in the number $n$ of lipid chains per molecule, which is consistent with the geometric shape transition of the lipopeptide micelles from spherical-like to flattened wormlike structures. Our simulations also reveal the bent conformations of the lipopeptide molecules in the self-assembled nanostructures which facilitate the close-packing of lipid chains in the micellar cores. The tilt angles between the peptide heads and the linked lipid chains show relatively wide distributions with average values in the range of $110^{\circ}$ to $140^{\circ}$. The di- and tri-lipidated lipopeptides are generally more extended than the mono-lipidated lipopeptides. Our simulation results on the microscopic structural features of the lipopeptide nanostructures may help to understand their bioactivities and provide useful information for guiding the design of bioactive medicines or drug carriers. The force fields built for these lipopeptides and the geometric packing model we proposed could also be applied for simulating and understanding the self-assembly behavior of other amphiphiles with similar chemical compositions.

As a final remark, the peptide sequences are found to be mainly taking disordered $\beta$ turn and random coil secondary structures, while the population of $\beta$-sheet structures is significantly lower than that reported in experiments at $300 \mathrm{~K}$. This discrepancy may arise from the fact that the secondary structures obtained at the simulation temperature of $300 \mathrm{~K}$ actually reflect the configurations at higher experimental temperature. This subject is left for future study.

\section{- ASSOCIATED CONTENT \\ Supporting Information}

The Supporting Information (SI) is available free of charge on the ACS Publications website at DOI: unknown.

There are four sections in the SI, including PS 1. Definitions of moment of inertia tensor, relative anisotropy shape and body reference frame; PS 2. Comparison of atomic density functions of different lipid chains in the same lipopeptide molecule; PS 3. Probability distributions of the length of lipid chains in lipopeptide molecules; PS 4. 
Probability distribution of angles (Including $\mathrm{Pam}_{3} \mathrm{CSK} 4$ molecules at the curved side edges of flattened wormlike micelle).

\section{- AUTHOR INFORMATION}

\section{Corresponding Authors}

*E-mail:ystu@yzu.edu.cn

*E-mail: zuowei.wang@reading.ac.uk

\section{ORCID}

Liang Zhao: 0000-0003-2687-1026

Yusong Tu: 0000-0002-7315-7901

Ian Hamley: 0000-0002-4549-0926

Zuowei Wang: 0000-0001-5759-9665

\section{Notes}

The authors declare no competing financial interest.

\section{- ACKNOWLEDGMENTS}

This work was supported by National Natural Science Foundation of China (Nos. 11605151, 11675138, 11422542), Shanghai Supercomputer Center of China and Special Program for Applied Research on Super Computation of the NSFC-Guangdong Joint Fund (the second phase). Z. Wang and I. Hamley acknowledge financial support from the Engineering and Physical Sciences Research Council (EPSRC) Platform Grant EP/L020599/1.

\section{REFERENCES}

(1) Pirri, G.; Giuliani, A.; Nicoletto, S. F.; Pizzuto, L. and Rinaldi, A. C., Lipopeptides as Anti-Infectives: a Practical Perspective. Cent. Eur. J. Biol. 2009, 4, 258-273.

(2) Wright, T. H.; Brooks, A. E.; Didsbury, A. J.; Williams, G. M.; Harris, P. W.; Dunbar, P. and Brimble, M. A., Direct Peptide Lipidation through Thiol-Ene Coupling Enables Rapid Synthesis and Evaluation of Self-Adjuvanting Vaccine Candidates. Angew. Chem. Int. Ed. 2013, 52, 10616-10619.

(3) Pretorius, D.; van Rooyen, J. and Clarke, K. G., Enhanced Production of Antifungal Lipopeptides by Bacillus Amyloliquefaciens for Biocontrol of Postharvest Disease. New Biotechnol. 2015, 32, 243-252.

(4) Webber, M. J.; Berns, E. J. and Stupp, S. I., Supramolecular Nanofibers of Peptide Amphiphiles for Medicine. Isr. J. Chem. 2013, 53, 530-554.

(5) Zhang, P.; Lu, J.; Huang, Y.; Zhao, W.; Zhang, Y.; Zhang, X.; Li, J.; Venkataramanan, R.; Gao, X. and Li, S., Design and Evaluation of a PEGylated Lipopeptide Equipped with Drug-Interactive Motifs as an Improved Drug Carrier. AAPS J. 2014, 16, 114-124.

(6) Bull, S. R.; Guler, M. O.; Bras, R. E.; Meade, T. J. and Stupp, S. I., Self-Assembled 
Peptide Amphiphile Nanofibers Conjugated to MRI Contrast Agents. Nano Lett. 2005, 5, 1-4.

(7) Hartgerink, J. D.; Beniash, E. and Stupp, S. I., Self-Assembly and Mineralization of Peptide-Amphiphile Nanofibers. Science 2001, 294, 1684-1688.

(8) Castelletto, V.; Gouveia, R. M.; Connon, C. J. and Hamley, I. W., New RGDPeptide Amphiphile Mixtures Containing a Negatively Charged Diluent. Faraday Discuss. 2013, 166, 381-397.

(9) Dehsorkhi, A.; Castelletto, V. and Hamley, I. W., Self-Assembling Amphiphilic Peptides. J. Pept. Sci. 2014, 20, 453-467.

(10) Castelletto, V.; Cheng, G.; Stain, C.; Connon, C. J. and Hamley, I. W., SelfAssembly of a Peptide Amphiphile Containing 1-Carnosine and Its Mixtures with a Multilamellar Vesicle Forming Lipid. Langmuir 2012, 28, 11599-11608.

(11) Cui, H.; Muraoka, T.; Cheetham, A. G. and Stupp, S. I., Self-Assembly of Giant Peptide Nanobelts. Nano Lett. 2009, 9, 945-951.

(12) Niece, K. L.; Hartgerink, J. D.; Donners, J. J. J. M. and Stupp, S. I., SelfAssembly Combining Two Bioactive Peptide-Amphiphile Molecules into Nanofibers by Electrostatic Attraction. J. Am. Chem. Soc. 2003, 125, 7146-7147.

(13) Shimada, T.; Sakamoto, N.; Motokawa, R.; Koizumi, S. and Tirrell, M., SelfAssembly Process of Peptide Amphiphile Worm-Like Micelles. J. Phys. Chem. B 2012, 116, 240-243.

(14) Niece, K. L.; Czeisler, C.; Sahni, V.; Tysseling-Mattiace, V.; Pashuck, E. T.; Kessler, J. A. and Stupp, S. I., Modification of Gelation Kinetics in Bioactive Peptide Amphiphiles. Biomaterials 2008, 29, 4501-4509.

(15) Tysseling-Mattiace, V. M.; Sahni, V.; Niece, K. L.; Birch, D.; Czeisler, C.; Fehlings, M. G.; Stupp, S. I. and Kessler, J. A., Self-Assembling Nanofibers Inhibit Glial Scar Formation and Promote Axon Elongation after Spinal Cord Injury. J. Neurosci. 2008, 28, 3814-3823.

(16) Kang, J. Y. and Lee, J. O., Structural Biology of the Toll-Like Receptor Family. Annu. Rev. Biochem. 2011, 80, 917-941.

(17) Hamley, I. W.; Kirkham, S.; Dehsorkhi, A.; Castelletto, V.; Reza, M. and Ruokolainen, J., Toll-Like Receptor Agonist Lipopeptides Self-Assemble into Distinct Nanostructures. Chem. Commun. 2014, 50, 15948-15951.

(18) Jin, M. S. and Lee, J. O., Structures of the Toll-Like Receptor Family and Its Ligand Complexes. Immunity 2008, 29, 182-191.

(19) Hamley, I. W., Lipopeptides: from Self-Assembly to Bioactivity. Chem. Commun. 
2015, 51, 8574-8583.

(20) Liu, K.; Wang, C.; Ma, J.; Shi, G.; Yao, X.; Fang, H.; Song, Y. and Wang, J., Janus Effect of Antifreeze Proteins on Ice Nucleation. Proc. Natl. Acad. Sci. U.S.A. 2016, 113, 14739.

(21) Yang, J.; Shi, G.; Tu, Y. and Fang, H., High Correlation between Oxidation Loci on Graphene Oxide. Angew. Chem. Int. Ed. 2014, 126, 10354-10358.

(22) Wan, R.; Wang, C.; Lei, X.; Zhou, G. and Fang, H., Enhancement of Water Evaporation on Solid Surfaces with Nanoscale Hydrophobic-Hydrophilic Patterns. Phys. Rev. Lett. 2015, 115, 195901.

(23) Shi, G.; Dang, Y.; Pan, T.; Liu, X.; Liu, H.; Li, S.; Zhang, L.; Zhao, H.; Li, S.; Han, J.; Tai, R.; Zhu, Y.; Li, J.; Ji, Q.; Mole, R. A.; Yu, D. and Fang, H., Unexpectedly Enhanced Solubility of Aromatic Amino Acids and Peptides in an Aqueous Solution of Divalent Transition-Metal Cations. Phys. Rev. Lett. 2016, 117, 238102.

(24) Tu, Y.; Lv, M.; Xiu, P.; Huynh, T.; Zhang, M.; Castelli, M.; Liu, Z.; Huang, Q.; Fan, C. and Fang, H., Destructive Extraction of Phospholipids from Escherichia Coli Membranes by Graphene Nanosheets. Nat. Nanotech. 2013, 8, 594-601.

(25) Liu, J.; Shi, G.; Guo, P.; Yang, J. and Fang, H., Blockage of Water Flow in Carbon Nanotubes by Ions Due to Interactions Between Cations and Aromatic Rings. Phys. Rev. Lett. 2015, 115, 164502.

(26) Zhao, L.; Wang, C.; Liu, J.; Wen, B.; Tu, Y.; Wang, Z. and Fang, H., Reversible State Transition in Nanoconfined Aqueous Solutions. Phys. Rev. Lett. 2014, 112, 078301.

(27) Tang, X.; Koenig, P. H. and Larson, R. G., Molecular Dynamics Simulations of Sodium Dodecyl Sulfate Micelles in Water-the Effect of the Force Field. J. Phys. Chem. B 2014, 118, 3864-3880.

(28) Fujiwara, S.; Itoh, T.; Hashimoto, M. and Horiuchi, R., Molecular Dynamics Simulation of Amphiphilic Molecules in Solution: Micelle Formation and Dynamic Coexistence. J. Chem. Phys. 2009, 130, 144901.

(29) Sangwai, A. V. and Sureshkumar, R., Binary Interactions and Salt-Induced Coalescence of Spherical Micelles of Cationic Surfactants from Molecular Dynamics Simulations. Langmuir 2012, 28, 1127-1135.

(30) Sammalkorpi, M.; Sanders, S.; Panagiotopoulos, A. Z.; Karttunen, M. and Haataja, M., Simulations of Micellization of Sodium Hexyl Sulfate. J. Phys. Chem. B 2011, 115, 1403-1410. 
(31) Sanders, S. A.; Sammalkorpi, M. and Panagiotopoulos, A. Z., Atomistic Simulations of Micellization of Sodium Hexyl, Heptyl, Octyl, and Nonyl Sulfates. J. Phys. Chem. B 2012, 116, 2430-2437.

(32) Velinova, M.; Sengupta, D.; Tadjer, A. V. and Marrink, S. J., Sphere-to-Rod Transitions of Nonionic Surfactant Micelles in Aqueous Solution Modeled by Molecular Dynamics Simulations. Langmuir 2011, 27, 14071-14077.

(33) Jusufi, A.; LeBard, D. N.; Levine, B. G. and Klein, M. L., Surfactant Concentration Effects on Micellar Properties. J. Phys. Chem. B 2012, 116, 987991.

(34) Wang, Z. and Larson, R. G., Molecular Dynamics Simulations of Threadlike Cetyltrimethylammonium Chloride Micelles: Effects of Sodium Chloride and Sodium Salicylate Salts. J. Phys. Chem. B 2009, 113, 13697-13710.

(35) Hargreaves, R.; Bowron, D. T. and Edler, K., Atomistic Structure of A Micelle in Solution Determined by Wide Q-Range Neutron Diffraction. J. Am. Chem. Soc. 2011, 133, 16524-16536.

(36) Sangwai, A. V. and Sureshkumar, R., Coarse-Grained Molecular Dynamics Simulations of the Sphere to Rod Transition in Surfactant Micelles. Langmuir 2011, 27, 6628-6638.

(37) Lee, O. S.; Stupp, S. I. and Schatz, G. C., Atomistic Molecular Dynamics Simulations of Peptide Amphiphile Self-Assembly into Cylindrical Nanofibers. J. Am. Chem. Soc. 2011, 133, 3677-3683.

(38) Martínez, L.; Andrade, R.; Birgin, E. G. and Martínez, J. M., PACKMOL: A Package for Building Initial Configurations for Molecular Dynamics Simulations. J. Comput. Chem. 2009, 30, 2157-2164.

(39) Hess, B.; Kutzner, C.; van der Spoel, D. and Lindahl, E., GROMACS 4: Algorithms for Highly Efficient, Load-Balanced, and Scalable Molecular Simulation. J. Chem. Theory Comput. 2008, 4, 435-447.

(40) Bussi, G.; Donadio, D. and Parrinello, M., Canonical Sampling through Velocity Rescaling. J. Chem. Phys. 2007, 126, 014101-014107.

(41) Parrinello, M. and Rahman, A., Polymorphic Transitions in Single Crystals: A New Molecular Dynamics Method. J. Appl. Phys. 1981, 52, 7182-7190.

(42) Miyamoto, S. and Kollman, P. A., Settle: An Analytical Version of the SHAKE and RATTLE Algorithm for Rigid Water Models. J. Comput. Chem. 1992, 13, 952-962.

(43) Darden, T.; York, D. and Pedersen, L., Particle Mesh Ewald: An N.log(N) Method 
for Ewald Sums in Large Systems. J. Chem. Phys. 1993, 98, 10089-10092.

(44) Essmann, U.; Perera, L.; Berkowitz, M. L.; Darden, T.; Lee, H. and Pedersen, L. G., A Smooth Particle Mesh Ewald Method. J. Chem. Phys. 1995, 103, 85778593.

(45) Oostenbrink, C.; Villa, A.; Mark, A. E. and Van Gunsteren, W. F., A Biomolecular Force Field Based on the Free Enthalpy of Hydration and Solvation: The GROMOS Force-Field Parameter Sets 53A5 and 53A6. J. Comput. Chem. 2004, 25, 1656-1676.

(46) Theodorou, D. N. and Suter, U. W., Shape of Unperturbed Linear Polymers: Polypropylene. Macromolecules 1985, 18, 1206-1214.

(47) Magnus Bergström, L., Second CMC in Surfactant Micellar Systems. Curr. Opin. Colloid. In. 2016, 22, 46-50.

(48) Bruce, C. D.; Berkowitz, M. L.; Perera, L. and Forbes, M. D. E., Molecular Dynamics Simulation of Sodium Dodecyl Sulfate Micelle in Water: Micellar Structural Characteristics and Counterion Distribution. J. Phys. Chem. B 2002, 106, 3788-3793.

(49) Israelachvili, J. N., Intermolecular and surface forces: revised third edition, Academic press, 2011.

(50) Heinig, M. and Frishman, D., STRIDE: A Web Server for Secondary Structure Assignment from Known Atomic Coordinates of Proteins. Nucleic Acids Res. 2004, 32, W500-W502.

(51) Leekumjorn, S. and Sum, A. K., Molecular Studies of the Gel to LiquidCrystalline Phase Transition for Fully Hydrated DPPC and DPPE Bilayers. BBA-Biomembranes 2007, 1768, 354-365. 
TOC Graphic

Flattened Wormlike Micelle

with a Bilayer Packing Structure

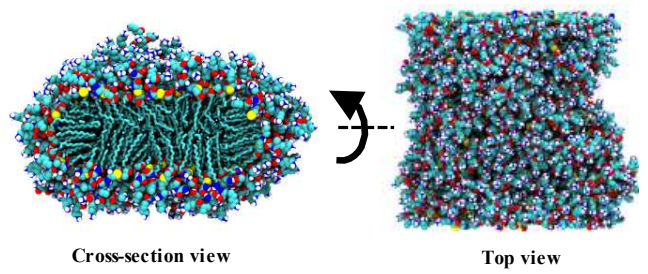

